\title{
Molecular Breeding Approaches for Drought and Drought Related Traits in Sorghum: A Review Article
}

\author{
Tamirat Bejiga*, Temesgen Teressa, Daniel Nadew \\ Ethiopian Institute of Agricultural Research (EIAR), Melkassa Agriculture Research Center, Adama, Ethiopia \\ *Corresponding Authors: Tamirat Bejiga, Ethiopian Institute of Agricultural Research (EIAR), \\ Melkassa Agriculture Research Center, Adama, Ethiopia
}

\begin{abstract}
Sorghum is one of the major important and multi-purpose cereal and more resilient crop to performs well under water constraints environment. Adaptation to harsh environments and crop with diverse end-uses makes sorghum a marvelous potential crop to meet the increasing demand of food security globally. However, under the variability and change of climate, drought is a major cause imposing yield reduction in sub-Saharan Africa countries in which the majority of farmers livelihoods depends on agriculture. Besides under rainfed condition potential of sorghum yield production affecting by terminal drought stress. Therefore, focusing on the drought tolerance genotype and drought tolerance related traits speed up the advancement of sorghum breeding program. This review update understanding of drought tolerant mechanisms, important drought related traits and breeding for drought tolerance of sorghum and genotype selection for moisture stress environments. The molecular breeding approaches provides unique benefits to researchers and scientists seeking to understand, characterize, and exploit agronomic traits and increase overall productivity of crops. That ultimately increase the availability of nutritious food and sustainable yield production. It can also serve as a fundamental breeding approaches to explicate the relationship among phenotypic and genotypic expression of crops and to create crops for upcoming needs of agricultural production.
\end{abstract}

Keywords: drought, sorghum, tolerance mechanism, molecular breeding, genetic engineering

\section{INTRODUCTION}

Sorghum [Sorghum bicolor (L.) Moench] is a photosynthetically efficient C4 grass, represents an essential source of grain, forage, fermentable sugars, and cellulosic fibers that can be utilized in myriad applications varying from bioenergy to bio industrial feedstocks (Boatwright et al., 2021).

Drought is a recurring phenomenon that puts crop yields production at risk and threatens the livelihoods of communities around the world (Liedtke et al., 2020). A grand challenge fronting agriculture is the advance of crop varieties with better drought resilience through breeding or biotechnology (Rajiv et al., 2020). Therefore, understanding the effect of drought on plants is critical for developing improved varieties with stable high yield. However, plant responses to drought stress are complex, depending on environmental conditions, frequency and duration of the stress, the species and variety of the plant, and the physiological stage of the plant at the time of the stress (Khan et al., 2016). To develop drought-resilient sorghum lines either through genetic engineering or molecular breeding is necessary to identify genes whose expression is associated with drought resistance about which little is known. Increased expression of rapidly induced transcription factors identified in drought-resistant genotypes in drought-sensitive genotypes through genetic engineering approaches could lead to the generation of drought-resistant cultivars (Salah et al., 2020).

For a successful breeding program, enough genetic diversity must be present in the starting population to find the right allelic combinations to enhance the resistance level through MAS or GS. New developments in biotechnology can create new sources of resistance and with potential to rapidly introgress in elite backgrounds (Khan et al., 2016).

Conventional breeding for improved grain production resulted in the development of improved cultivars with significant increase in the productivity of crop. However, several biotic and abiotic factors such as drought, insect pests, fungal pathogens and parasitic weed like striga could not be combated efficiently through conventional breeding techniques alone (Girijashankar and Swathisree, 2009). Advances in marker-assisted selection, in vitro cellular techniques and recombinant DNA 
technologies are underpinning major innovations in plant breeding. The full range of biotechnologies, including genome editing is well placed to play a significant part in meeting the challenge of global food security (Jones, 2016).

In recent years, plant biotechnology, including molecular genetics and genomics as well as plant transformation, has provided a powerful means to supplement traditional breeding approaches. The high variation among the sorghum genotypes was distinguished by using ISSR markers which demonstrating that, the technique was resourceful in determining the genetic diversity among sorghum genotypes (Nada et al., 2016). Also, the SSR data are useful in identifying genetic relationships among genotypes. The clustering generated by the molecular markers, combined with the drought tolerance information, can be useful in selecting parents for crossing to breed drought tolerant varieties (Ahmed et al., 2009).

In most breeding programmes, the genetic enhancement for drought resistance is achieved through selection for yield and because of low heritability of yield under stress and the spatial as well as timebased variation in the field environment, conventional breeding approaches are slow. Whereas molecular markers such as restriction fragment length polymorphism (RFLP), random amplified polymorphic DNA (RAPD) and isozyme will facilitate development of drought-resistant genotypes more effectively as their expressions are independent of environmental effects. Subsequently identification of the molecular markers linked with yield or other morphological traits related to drought resistance, those markers could be used as selection criteria for drought resistance. The application of marker assisted selection in evolving drought resistant genotypes is in an experimental stage; more specifically just identification of RFLP markers associated with osmotic adjustment, stay green, root traits have been achieved (Mc Couch CL et al., 1998).

The empowerment of standardized protocols for gene transformation and regeneration in sorghum opens up new opportunities to improve protein nutritional quality, high yield and drought resistant cultivars which serve as an ideal staple food for ever increasing population. The ultimate aims of genetic transformation studies are to develop user friendly vector system applicable to a wide range of species (Darika et al., 2013). Besides genomics has opened up new perspectives and opportunities for marker assisted selection for plant breeders, to assess and enhance diversity in their germplasm collections, to introgress valuable traits from new sources and to identify genes that control key traits (Sharmila et al., 2014). The ability to manipulate drought tolerance through marker-assisted backcrossing makes it much simpler to introduce such a new trait into a breeding program (Kassahun et al., 2010).

Crop genome sequencing projects generate enormous amounts of genomic sequence information, and the utilization of this information in applied crop improvement programs has been augmented by the availability of sophisticated bioinformatics tools. Based on sequence homology, we aligned all publicly available simple sequence repeat markers on a sequence-based physical map for sorghum (Ramu et al., 2010). The genomic regions enriched with heat shock protein (HSP), expansin, and aquaporin genes responsive to drought stress could be used as powerful targets for improvement of drought tolerance in sorghum and other cereals (Zhang et al., 2019).

Understanding the molecular response of crops to drought is critical to determinations to enhance agricultural yields under progressively frequent droughts (Varoquauxa et al., 2019). The aim of this review to investigate sorghum molecular breeding aspect, important drought related traits and its roles in drought tolerance.

\section{SORGHUM GENETIC DIVERSITY}

Sorghum is further improved and diversified into diverse end uses through breeding selection. The use of sorghum varies significantly amongst different regions of the word (Figure 1). Generally, it is mainly used for food in developing countries and for feed in developed countries (Huaiqing et al., 2021). Due to its extensive genetic diversity and worldwide colonization, sorghum has considerable diversity for a range of phenotypes influencing productivity, composition, and sink/source dynamics (Boatwright et al., 2021).

The eastern Africa region, Ethiopia and its surroundings, is considered as the center of origin and diversity for sorghum, and has contributed to global sorghum genetic enhancement (Girma et al., 2019). As Cuevas et al., 2017 reported that the Ethiopian germplasm genetic diversity is largely 
unused in sorghum breeding programs, this indicating sorghum breeders and geneticists worldwide with knowledge and tools to utilize and conserve this germplasm. For the fact that Sorghum is one of an indigenous crop of Ethiopia, there are tremendous amount of variability exists in the country (Adugna, 2007). Sorghum germplasm from Ethiopia is one of the richest for characters diversity. This diversity has evolved under the diverse and unique environmental conditions and also selected by farmers for diverse uses. The core of genetic and genomic resources is the availability and accessibility of germplasm to relation genotype to phenotype (Richard et al., 2019). Ethiopian farmers mostly grow sorghums with colored pericarps as they consider them better in nutritional values and are better tolerant to biotic and abiotic stresses (Girma et al., 2019).

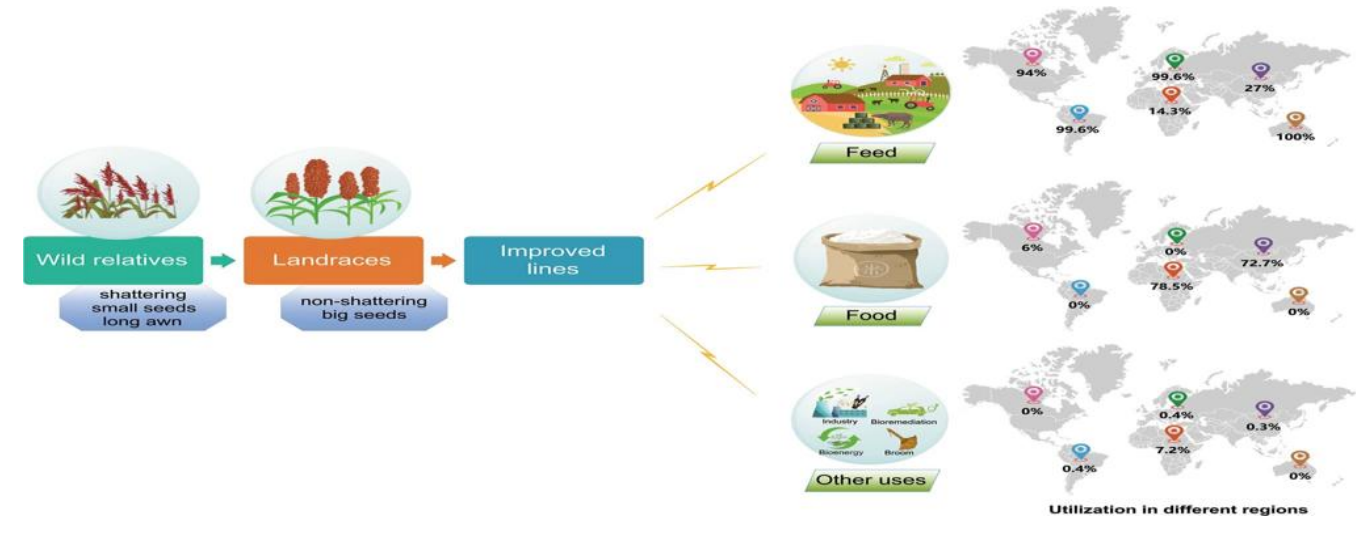

Source: Huaiqing et al., 2021

Figure1. A diagram indicated the remarkable feature of sorghum as a multiple-purpose crop. The National Plant Germplasm System (NPGS) of Ethiopian germplasm collection is the major group of accessions in the sorghum collection. The Ethiopian NPGS germplasm collection is a greatly genetically and phenotypically various. Untapping the genetic variation is needs precise strategies for sorghum enhancement. The use of molecular markers associated with important agronomic traits could facilitate the identification of valuable accessions (Cuevas et al., 2017).

The phenotypic characterization of populations is necessary to intensely understand the population structure and advance the use of the collection (Cuevas et al., 2017). The genetic architecture of the complex Ethiopian sorghum germplasm was established and the characterization of genes and alleles controlling agronomic traits contributes as a source of markers for molecular breeding (Girma et al., 2019). The development of new cultivars with novel alleles is a promising strategy to translate genetic diversity into agriculture benefits (Cuevas et al., 2017).

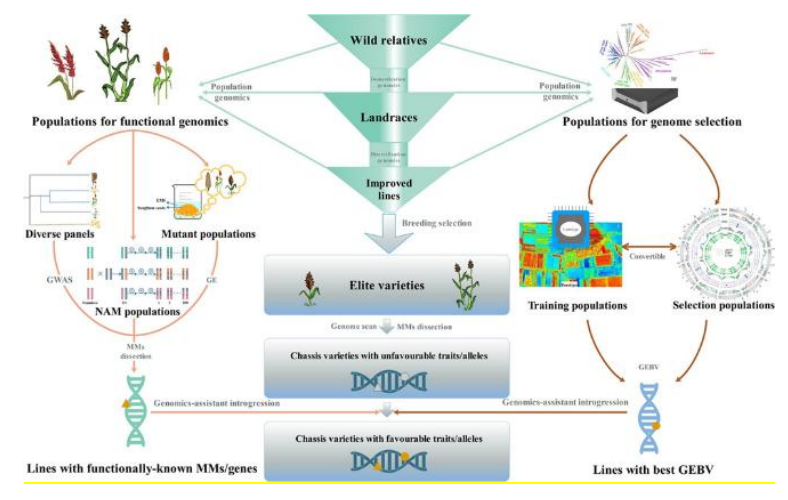

Source: Huaiqing et al., 2021

Figure2. Breeding scheme for sorghum improvement using the state-of the-art genomics-based breeding strategies.

The breeding programme consists of four key components. Part I: Diagnosis of changes in genetic diversity during domestication and diversification; Part II: Discovery and characterisation of genetic and genomic variation; Part III: Selection of pre-breeding materials through genome selection; Part IV: Genomics-assistant introgression and improvement in elite varieties (Huaiqing et al., 2021). 


\section{BREEDING FOR DROUGHT RESISTANCE}

Drought is a major threat to food production worldwide and its impact is only expected to increase with the further onset of climate change (Khan et al., 2016). Increasing water use efficiency under climate change will result from two fronts. First, being able to identify genotypes that have high assimilation rates under temperature and water-deficit stress. Second, we need to realize that there are a range of management practices we can adopt that will reduce soil water evaporation and shift the water use by the crop to more transpiration to limit the exposure of the plant to water-deficit stress and maintain productivity at the highest level possible (Hatfield and Dold, 2019). Drought is occurred by high temperature, low water potential, low humidity and reduced availability of soil moisture during pre and post flowering crop grow phase. Avoidance, tolerance and escape are major mechanisms of crop to grown in drought prone environments. Escape mechanism includes early flowering, early maturity, high leaf nitrogen level, high photosynthetic capacity andremobilization of assimilates.

A tolerance mechanism includes osmotic adjustments, protective solutes, high proline, desiccation tolerant enzymes, and high stomatal conductance. Avoidance mechanism of crop includes deep root, stomatal closure, leaf rolling, tissue hydration, stem waxiness, stay-green and hightranspiration efficiency (Ashok et al., 2018). Currently, plant biotechnology is one of the most hopeful fields for developing crops that are able to produce high yields in moisture stress conditions. Most plants with improved drought resistance have demonstrated decreased crop yield, meaning that there is still a requirement to search for novel approaches that can uncouple drought resistance from plant growth (Martignago et al., 2020). In response to drought, plants reprogram their gene expression, which ultimately regulates a multitude of biochemical and physiological processes. The timing of this reprogramming and the nature of the drought-regulated genes in different genotypes are thought to confer differential tolerance to drought stress. Sorghum is a highly droughttolerant crop and has been increasingly used as a model cereal to identify genes that confer tolerance (Salah et al., 2020).

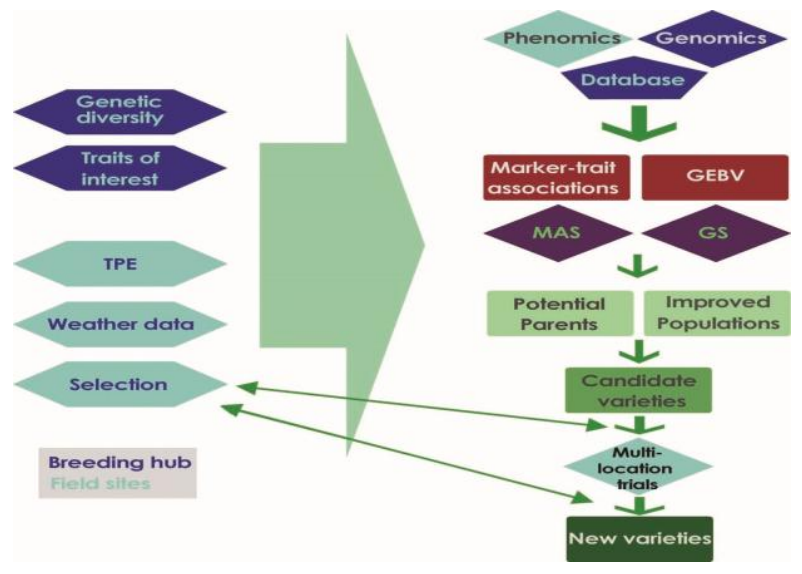

Source: Khan et al., 2016

Figure3: The complex nature of drought tolerance needs a multipronged approach to develop new varieties with stable and enhanced yield under stress conditions.

High throughput genomics and phenomics offer rapid and targeted drought-tolerant varieties and genetic gains via Marker-assisted selection (MAS) and genomic selection (GS). The breeding program should be split between a breeding hub (shapes in dark blue) for centralized activities such as genotyping, definition of traits of interest, and maintenance of genetic diversity, databases, and analytical tools, and field sites (shapes in light blue) with well-characterized stress scenarios, weather data, and soil analysis for targeted-site specific phenotyping and stress trials. In this figure, hexagons stand for data required as an input, diamonds are steps to be performed, and rectangles are outputs (Khan et al., 2016).

\section{Important sorghum Traits to Drought tolerance}

Drought tolerance refers to the ability of plants to withstand shortage of water while maintaining appropriate physiological activities to stabilize and protect cellular and metabolic integrity at tissue 
and cellular level (Xiong et al., 2006; Tuinstra et al., 1997). Survival is the ability of the crop to survive drought, irrespective of the yield it produces, while production is the ability of the crop to grow and yield under water stress conditions (Beyene et al., 2015). The important mechanisms in drought tolerance are including osmotic adjustments, stay green, leaf rolling, waxy ness on stem, root morphology and its architecture, transpiration efficiency and secretion of soluble solutes (Ashok et al., 2018).

\section{Early Flowering and Drought Escape}

Drought escape is a classical adaptive mechanism to enable plant to complete full life-cycle prior to a coming drought occurred. Early flowering time and shorter vegetative are important traits in conditions of terminal drought that can minimize exposure to dehydration during the sensitive flowering and post-anthesis grain filling periods (Shavrukov et al., 2017). Drought stress during flowering will reduce silk elongation rate, increase the anthesis silking interval and result in reduced ovule fertilization and increased kernel abortion. Therefore, flowering before the onset of growth limiting drought is an important way to ensure yield formation in environmental conditions with insecure rainfall/ terminal drought. In the past selection for drought tolerance has been carried out for improved harvest index, stay green and shorter ASI (Edmeades et al., 2000). The pre-flowering tolerant genotype RTx430 and for post flowering tolerant genotype BTx642 had fewer genes differentially expressed in the leaf after a week of drought exposure. Genotype-specific adaptationto pre-flowering and post-flowering moisture stress mainly determined by attempts to maintain normal activity in leaves (Varoquauxa et al., 2019). The maximum detrimental drought stress is that which happens during the post-flowering stage of crop growth (Kassahun et al., 2010)

Source: Ashok et al., 2018

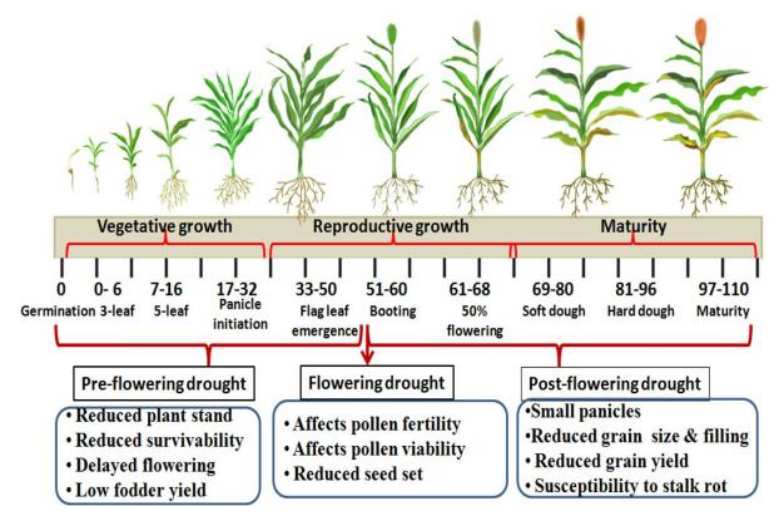

Figure4. Different growth stages of sorghum and effect of Pre and post flowering drought stress on various sorghum agronomic traits.

\section{LEAF AREA}

Leaves subjected to drought stress show variable responses in water use efficiency. The response of water use efficiency at the leaf level is directly related to the physiological processes controlling the gradients of $\mathrm{CO} 2$ and $\mathrm{H} 2 \mathrm{O}$ between the leaf and air surrounding the leaf (Hatfield and Dold, 2019). Leaf area had important role in drought tolerance of sorghum. In drought prone areas leaf area of sorghum is smaller for save loss of water through evapotranspiration. Under drought environment optimum leaf area is vital for optimum photosynthetic activity. Besides, optimum leaf area is important for producing high dry matter as well as grain yield in drought areas (Tesfamichael et al., 2015).

Water stress decreased leaf area index, leaf area duration, crop growth rate and tillers per plant. Fazeli, 2015 research result shown that the applied SAP had an important effect on forage sorghum and increased leaf area index, leaf area duration, crop growth rate and tillers per plant. Probably the application of SAP could be an effective management practice in soils characterized by low water holding capacity where irrigation water and fertilizer often leach below the root zone within a short period of time, leading to poor water and fertilizer use efficiency by crops. Therefore, SAP increases leaf area index through increasing both water and nutrient use efficiency in crops. The higher LAI 
causes an increase in LAD and results in increasing available assimilate in the plant. The increasing available assimilate caused increasing in crop growth rate (Fazeli, 2015). The dynamics of crop water use and biomass accumulation have to consider soil water evaporation rate, transpiration from the leaves, and the growth pattern of the crop. Enhancing water use efficiency at the canopy level can be achieved by adopting practices that reduce the soil water evaporation component and divert more water into transpiration (Hatfield and Dold, 2019).

As Alemu et al., 2020 reported that leaf area showed a significant negative association with root length and root angle, however significant positive association to stem diameter, shoot fresh weight, shoot dry weight and root fresh weight. In order to increase fresh and dry weight of shoot, the breeders must select germplasm having narrow root angle.

\section{Stay Green}

Stay-green is a moisture stress adaption phenotype found in sorghum and other cereals. Plants expressing stay green show maintain functional green leaves for longer when moisture stress occurs during grain fill (Liedtke et al., 2020). In grain sorghum breeding grain yield and stay-green are target traits in selection for broad adaptation range of environments (Velazco et al., 2019).

Stay-green has been exhibited to enhance tolerance of post flowering drought in grain sorghum. In sorghum a number of QTLs associated with stay green have been identified, therefore facilitating transfer of this trait into adapted genetic backgrounds is important (Kassahun et al., 2010).

Liedtke et al., (2020) reported pre flowering canopy parameters was weakly correlated with staygreen values for leaf senescence whereas post flowering canopy parameters exhibited a much stronger association with leaf senescence. This result indicated that canopy size before flowering made a relatively small role to the expression of a stay-green phenotype after flowering. The resistance mechanisms exhibited by the studied varieties were acceleration of their leaf senescence to decrease water loss and the increase of their roots length density to explore the deeper soil layers moisture (Gano et al., 2021)

\section{Stomatal-Mediated DROUGHT RESPONSES}

At the leaf level, water use is controlled by the available energy impinging on the leaf, vapor pressure deficit, and aerodynamic exchange, but, regulated by stomatal conductance. While at the canopy level, the processes involve energy exchange at the soil surface and the plant canopy and water loss is a combination of evaporation from the soil surface and transpiration from the plant canopy (Hatfield and Dold, 2019). The varieties physiologically responded to early water scarcity by closing the stomata and then decreasing leaf transpiration and photosynthesis rate (Gano et al., 2021).

\section{Cuticular Wax Production}

Cuticular wax is first protective layer of above ground tissues of many plant species and plays significant roles in plant abiotic and biotic stress tolerance and has been implicated in defense mechanisms against excessive ultraviolet radiation, high temperature, bacterial and fungal pathogens, insects, high salinity, and low temperature. (Xue et al., 2017). Sorghum accumulates epi-cuticular wax (EW) in leaves, sheaths, and culms. Epi-cuticular wax reduces the transpirational and nontranspirational (nonstomatal) water loss and protects the plant from severe drought stress in addition to imparting resistance against insect pests (Elango et al., 2020)

Modern research findings have revealed that small RNAs (sRNAs) such as microRNA and smallinterfering RNA play important roles in plant stress adaptation. In addition, leaf cuticle and surface wax serve as an important trait for stress tolerance, and many regulatory genes coordinate wax accumulation. (Sajeevan et al., 2017).

\section{Root Traits}

Roots are the plant organ responsible for water and nutrient uptake and interaction with soil microbes and play a crucial role in moisture stress tolerance (Ruben et al., 2020). Root traits related with maintaining plant productivity under moisture stress areas include small fine root diameters, long specific root length, and root length density at depths in soil with available water (Comas et al., 2013).

Phenotyping root is one of the drought management tools. Roots play a significant role in the plant's life by extracting soil resources from deeper soil layers and its phenotyping helps to understand 
different root traits (Allah et al., 2018). Identified QTL landraces as a source of favorable root traits to introgress into adapted phenotypes through marker-assisted (Ruben et al., 2020).

Sorghum access to extra water through mechanisms of deeper roots (Liedtke et al., 2020). In drought environments small xylem diameters in targeted seminal roots save soil water deep in the soil profile for use during crop maturation and result in improved yields. Capacity for deep root growth and large xylem diameters in deep roots may also improve root acquisition of water when ample water at depth is available (Comas et al., 2013).

Root's demonstration more transcriptional interruption than leaves. While both leaf and root samples exhibited an extensive response to drought, root samples indicated both a larger number of differentially stated genes, as well as a larger absolute change in expression of those genes, related to leaf samples (Varoquauxa et al., 2019). There was not a large difference in the number of transcriptional changes between the pre flowering drought tolerant genotype RTx430 and post flowering drought tolerant genotype BTx6422 genotypes in the root after a week of drought exposure. These indicated the root is more substantially affected total by moisture stres

(Varoquauxa et al., 2019). Under moisture stress condition smaller diameter roots, greater SRL, and increased root hair density or length should improve plant acquisition of water and reduce plant carbon investment required for that acquisition. In addition, crop hydraulic functioning under moisture stress may be improved through enhanced capacity for nocturnal refilling of embolized xylem and changes in inter-vessel pit anatomy to reduce cavitation (Comas et al., 2013). The identification of molecular markers linked to root system architecture (RSA) traits is special interest to the breeding community (Ruben et al., 2020).

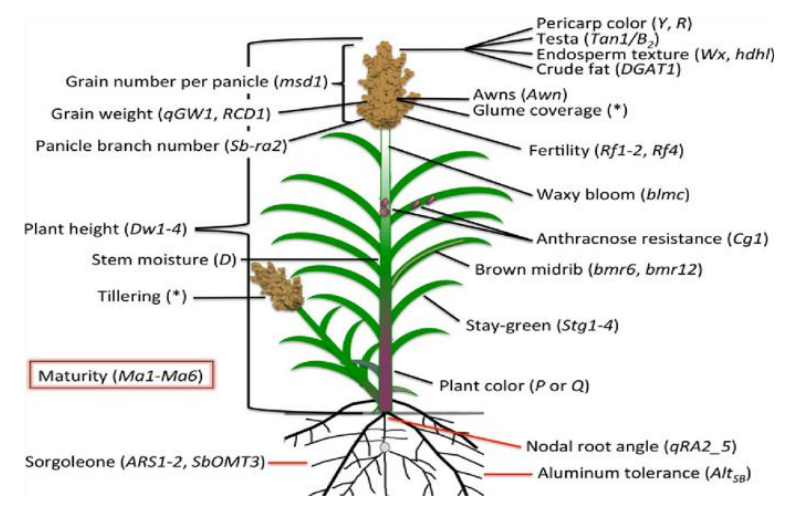

Source: Richard et al., 2019.

Figure5. The majority of major effect quantitative trait locus $(Q T L) / g e n e s$ projected onto a sorghum animation.

Asterisks denote traits with multiple QTLs that have yet to be characterized and/or fine-mapped.

\section{MOLECUlar Markers}

Sorghum is a cereal crop with distinctive drought resilience and is an unused resource of allelic diversity (Rajiv et al., 2020). A molecular marker a DNA sequence that is readily detected and whose inheritance can be easily be monitored. The uses of molecular markers are based on the naturally occurring DNA polymorphism, which forms basis for designing strategies to exploit for applied purposes. A marker must to be polymorphic, it must exit in different forms so that chromosome carrying the mutant genes can be distinguished from the chromosomes with the normal gene by a marker it also carries. Genetic polymorphism is defined as the simultaneous occurrence of a trait in the same population of two discontinuous variants or genotypes. DNA markers seem to be the best candidates for efficient evaluation and selection of plant material. Unlike protein markers, DNA markers segregate as single genes and they are not affected by the environment. DNA is easily extracted from plant materials and its analysis can be cost and labour effective. The first such DNA markers to be utilized were fragments produced by restriction digestion the restriction fragment length polymorphism (RFLP) based genes marker. Consequently, several markers system has been developed (Kumar et al., 2009). 
Marker assisted selection can reduce the time required for line development. This is due to the fact that this method does not require progeny test for each cycle of backcrossing. Moreover, this method also reduces costs for labor and progeny trials, and it may also reduce population size for line selection. Marker assisted selection requires well-equipped laboratory and the cost for line development may be higher than conventional method. However, the method is faster, leading to the rapid release of new cultivars (Darika et al., 2013).
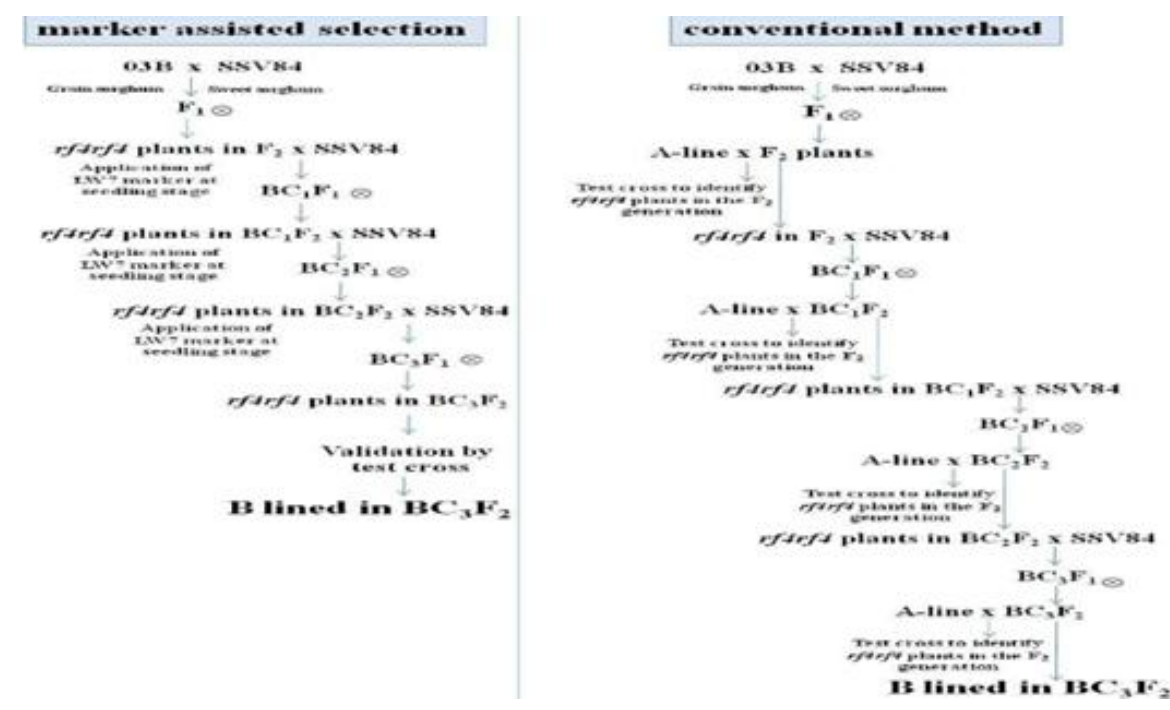

Source: Darika et al., 2013

Figure6. Schematic diagrams of marker assisted selection and conventional selection method.

Marker-assisted backcrossing effectiveness in introgression programs (Kassahun et al., 2010). As a consequence of permitting simultaneous foreground selection for introgression of an exotic allele with potential to improve performance for a trait of attention and background selection for the preferred recurrent parent genotype in other genomic regions.

Integration of the sorghum genetic map developed from QTL information with the physical map will greatly facilitate the map based cloning and precise dissection of complex traits such as drought tolerance in sorghum. More research is needed in the area of sorghum DNA based maps for identifying and characterizing genes of interest. Tools of biotechnology provide great potential for the exploitation of untapped germplasm of sorghum. The research advances in genomics will greatly accelerate the acquisition of knowledge with further development of tools for modifying and interrogating genomes. ICRISAT developed diversification of sorghum breeding programs by the incorporation of new traits and genetic materials. An effort to insert pest resistance in sorghum seems to be successful for shoot fly and Midge (Sharmila et al., 2014).

\section{GENETIC ENGINEERING FOR DROUGHT TOLERANT TRAITS}

The use of genetic engineering techniques has high hopes for the production of transgenic plants with no or little effect on plants physiological and morpho biochemical properties. Most of the genes reported to be related with drought tolerance also confer resistance, to some extent, to other environmental stresses (Rida et al., 2020). Genetic transformation is a powerful tool for genetic improvement of arable crops.

Progress in sorghum transformation has been hampered by difficulties associated with tissue culture, such as accumulation of phenolic pigments and low regeneration frequencies. The long periods of selection needed for the recovery and regeneration of putative transgenic plants often hampered optimization of conditions for sorghum transformation. Probably, low transferability of sorghum was predicted as occurrence of DNA methylation in sorghum cells that inactivates the expression of transferred genes (Sharmila et al., 2014). Genetic engineering approaches are especially important for modification of starch and protein contents, vitamin and micronutrient concentration, improvement of nutritive value of protein fractions, and increase tolerance to environmental stresses (Elkonin et al., 2012). 
Plant transformation is performed using a wide range of techniques such as Agrobacterium Ti plasmid vectors, micro projectile bombardment, electroporation, microinjection, chemical (PEG) treatment of protoplasts. Transformation using Agrobacterium and micro projectile bombardment are currently the most extensively used approaches. Agrobacterium-based DNA transfer system offers many unique advantages in plant transformation: (1) the simplicity of Agrobacterium gene transfer makes it a poor man's vector. (2) A precise transfer and integration of DNA sequences with defined ends. (3) Linked transfer of genes of interest along with the transformation marker. (4) The higher frequency of stable transformation with single copy insertions. (5) Reasonably low incidence of transgene silencing. (6) The ability to transfer long stretches of T-DNA. Genetic transformation of crop species using Agrobacterium is believed to be more practical, as the success rates of transformation are greater than biolistic approach. Further, unlike later, complex equipment is not involved. However, for a long period of time monocotyledons have been considered outside the host range of Agrobacterium. But, advances in understanding the biology of the infection process and the availability of suitable gene promoters as well as selectable markers improved transformation in monocotyledons (Girijashankar and Swathisree, 2009).

To enhance the tolerance to water deficit and $\mathrm{NaCl}$ stress, the mtlD gene encoding for mannitol1phosphate dehydrogenase from E. coli was used for sorghum transformation (Maheswari et al., 2010). The improved drought tolerance of transgenic sorghum was illustrated by the increased retention of leaf water. Moreover, there was a significantly improved maintenance in root and shoot growth of transformed plants under $\mathrm{NaCl}$ stress.

\section{CONCLUSION}

Sorghum is a multipurpose staple crop and the species shows greater diversity. Previously, improvement of sorghum has been carried out mostly using traditional plant breeding methods, but this method is less realistic. Currently, advances in biotechnology and genetic transformation are used commonly to assist traditional breeding for crop improvement.

Drought is one of the major abiotic constraints to reduced or total loss sorghum grain yield production in dry lowland environments. For that reason, improving the drought tolerance of sorghum is required focus and research areas of plant breeders, to minimize the yield losses resulting from drought stress.

Sorghum engineering frequency has increased significantly due to improvements in tissue culture and transformation conditions. Genetic transformation offers direct access to a vast pool of useful genes not previously accessible to plant breeders. Among the DNA-delivery methods that have been utilized for sorghum transformation, the bombardment and Agrobacterium-mediated methods are the most efficient. Genome sequencing, together with discovery of candidate genes and promoters, will continue to be very useful for sorghum genetic engineering. These new genetic resources provide opportunities to develop sorghum varieties with important traits required for food consumption and biotic and abiotic resistance. This review contributes to the understanding of the molecular breeding approaches of sorghum for drought adaptation and provides hints for identifying key mechanisms of drought tolerant and related traits important for sorghum enhancement in drought tolerance.

\section{REFERENCES}

[1] Adugna Asfaw, 2007. The role of introduced sorghum and millets in Ethiopian agriculture Melkassa Agricultural Research Center, Nazareth, Ethiopia. SAT Journal ejournal.icrisat.org 3 (1).

[2] Ahmed H. Abu Assar, Ralf Uptmoor, Awadalla A. Abdelmula, Carolla Wagner, Mohammed Salih, Abdelbagi M. Ali, Frank Ordon and Wolfgang Friedt, 2009. Assessment of Sorghum Genetic Resources for Genetic Diversity and Drought Tolerance Using Molecular Markers and Agro-morphological Traits. U. of K. J. Agric. Sci. 17(1):1-22).

[3] Alemu Tebeje, Kassahun Bantte, Temesgen Matiwos and Andrew Borrell, 2020. Characterization and association mapping for drought adaptation in Ethiopian sorghum (Sorghum bicolor (L.) Moench) germplasm. Vegetos https://doi.org/10.1007/s42535-020-00163-0

[4] Allah Wasaya, Xiying Zhang, Qin Fang and Zongzheng Yan, 2018. Root Phenotyping for Drought Tolerance: A Review. Agronomy 8:241; doi:10.3390/agronomy8110241

[5] Ashok Badigannavar, Niaba Teme, Antonio Costa de Oliveira, Guying Li, Michel Vaksmann, Vivian Ebeling Viana, T. R. Ganapathi and Fatma Sarsu, 2018. Physiological, genetic and molecular basis of 
drought resilience in sorghum [Sorghum bicolor (L.) Moench]. Indian Journal for Plant Physiology. https://doi.org/10.1007/s40502-018-0416-2

[6] Beyene, A., Hussien, S., Tongoona, P., and Liang, M. (2015). Physiological mechanisms of drought tolerance in sorghum, genetic basis and breeding methods: A review. African Journal of Agricultural Research 10: 3029-3040.

[7] Boatwright J. Lucas, Brenton Zachary W., Boyles Richard E., Sirjan Sapkota, Myers Matthew T., Jordan Kathleen E., Dale Savanah M., Nadia Shakoor, Cooper Elizabeth A., Morris Geoffrey P. and Stephen Kresovich, 2021. Genetic Characterization of a Sorghum bicolor Multiparent Mapping Population Emphasizing Carbon-Partitioning Dynamics. Genetics Society of America 1-14

[8] Comas Louise H., Becker Steven R., Cruz Von Mark V., Byrne Patrick F. and Dierig David A., 2013. Root traits contributing to plant productivity under drought. Frontiers in Plant Science 4:442 doi:10.3389/fpls.2013.00442

[9] Cuevas, H.E., Rosa-Valentin, G., Hayes, C.M., Rooney, W.L., Hoffmann, L. 2017. Genomic characterization of a core set of the USDA-NPGS Ethiopian sorghum germplasm collection: implications for germplasm conservation, evaluation, and utilization in crop improvement. BMC Genomics. 18:108. doi:10.1186/s12864-016-3475-7.

[10] Darika Bunphan, Prasit Jaisil and Jirawat Sanitchon, 2013. Application of LW7 marker for identification of progenies with male sterility gene in sweet sorghum population. Crop Breeding and Applied Biotechnology 13: 59-66.

[11]Edmeades GO, Bolanos J, Elings A, Ribaut JM, Banziger M, Westgate ME, 2000. The role and regulation of the anthesis-silking interval in maize, p. 43-73, In Volenec J., ed. Physiology and Modeling Kernel Set in Maize.

[12] Elango, D., Xue, W. \& Chopra, S., 2020. Genome wide association mapping of epi-cuticular wax genes in Sorghum bicolor. Physiol Mol Biol Plants 26, 1727-1737. https://doi.org/10.1007/s12298-020-00848-5

[13]Elkonin L.A., O.N. Nosova and J.V. Italianskaya, 2012. Agrobacterium-Mediated Genetic Transformation of Sorghum Using Tissue Culture-Based and Pollen Mediated Approaches. Journal of Stress Physiology \& Biochemistry, Vol. 8 No. 3.

[14]Fazeli Rostampour M., 2015. Relationship between Some Growth Indexes and Tillering of Forage Sorghum under Irrigation Regimes and Polymer. Journal of Biotechnology Research Vol. 1 pp: 1-7.

[15]Gano, B.; Dembele, J.S.B.; Tovignan, T.K.; Sine, B.; Vadez, V.; Diouf, D.; Audebert, A., 2021. Adaptation Responses to Early Drought Stress of West Africa Sorghum Varieties. Agronomy 11(443):1-21. https:// doi.org/10.3390/agronomy11030443

[16] Girijashankar V. and Swathisree V., 2009. Genetic transformation of Sorghum bicolor Physiol. Mol. Biol. Plants, 15(4).

[17] Girma G, Nida H, Seyoum A, Mekonen M, Nega A, Lule D, Dessalegn K, Bekele A, Gebreyohannes A, Adeyanju A, Tirfessa A, Ayana G, Taddese T, Mekbib F, Belete K, Tesso T, Ejeta G and Mengiste T., 2019. A Large-Scale Genome-Wide Association Analyses of Ethiopian Sorghum Landrace Collection Reveal Loci Associated with Important Traits. Front. Plant Sci. 10:691. doi: 10.3389/fpls.2019.00691

[18] Hatfield JL and Dold C., 2019. Water-Use Efficiency: Advances and Challenges in a Changing Climate. Front. Plant Sci. 10:103. doi: 10.3389/fpls.2019.00103

[19] Huaiqing Hao, Zhigang Li, huanyuan Leng, Cheng Lu, Hong Luo, Yuanming Liu, Xiaoyuan Wu, Zhiquan Liu, Li Shang and Hai-Chun Jing, 2021. Sorghum breeding in the genomic era: opportunities and challenges. Theoretical and Applied Genetics. https://doi.org/10.1007/s00122-021-03789-z

[20] Jones H.D., 2016. Introduction: Biotechnology of Major Cereals. CAB International. PP 1-4.

[21] Kassahun Bante, F. R. Bidinger, C. T. Hash and M. S. Kuruvinashetti, 2010. Stay-green expression in early generation sorghum [Sorghum bicolor (L.) Moench] QTL introgression lines. Euphytica 172:351-362. DOI 10.1007/s10681-009-0108-0

[22] Khan Awais, Valpuri Sovero and Dorcus Gemenet, 2016. Genome-assisted Breeding for Drought Resistance. Current Genomics 17(4): 330-342.

[23] Kumar P., Gupta V.K., Misra A.K., Modi D.R. and Pandey B.K., 2009. Potential of Molecular Markers in Plant Biotechnology. Plant Omics Journal 2(4):141-162. 
[24]Liedtke J. D., C. H. Hunt, B. George-Jaeggli, K. Laws, J. Watson, A. B. Potgieter, A. Cruickshank and D. R. Jordan, 2020. High-Throughput Phenotyping of Dynamic Canopy Traits Associated with Stay-Green in Grain Sorghum. Plant Phenomics. https://doi.org/10.34133/2020/4635153

[25] Maheswari M, Varalaxmi Y, Vijayalakshmi A, Yadav SK, Sharmila P, Venkateswarlu B, Vanaja M, Saradhi PP, 2010. Metabolic engineering using $m t l D$ gene enhances tolerance to water deficit and salinity in sorghum. Biol Plantarum 54:647-652.

[26] Martignago D, Rico-Medina A, Blasco-Escámez D, Fontanet-Manzaneque JB and Caño-Delgado AI, 2020. Drought Resistance by Engineering Plant Tissue-Specific Responses. Front. Plant Science 10:1676. https://doi.org/10.3389/fpls.2019.01676

[27] Mc Couch CL, Xiao J., 1998. In molecular dissection of complex traits. In:(ed. Paterson AH), CRC Press, Boca Raton.

[28] Nada B. Hamza, Atif E. Idris, Ismael I. Elmunsor, Ali I.A. Ibrahim and Atif I. Abuali, 2016. Drought tolerance assessment in grain sorghum (Sorghum bicolor [L.] Moench) genotypes using agro-morphological traits and DNA markers. International Journal of Plant Breeding and Genetics, 10 (3): 125-131. DOI: 10.3923/ijpbg.2016.125.131.

[29] Rajiv K. Parvathanenia, Indrajit Kumara, Max Brauda and Andrea L. Evelanda, 2020. Regulatory signatures of drought response in stress resilient Sorghum bicolor. https://doi.org/10.1101/2020.08.07.240580

[30] Ramu, P., Deshpande, S.P., Senthilvel, S. et al. 2010. In silico mapping of important genes and markers available in the public domain for efficient sorghum breeding. Mol Breeding 26, 409-418. https://doi.org/10.1007/s11032-009-9365-9).

[31] Richard E. Boyles, Zachary W. Brenton and Stephen Kresovich, 2019. Genetic and genomic resources of sorghum to connect genotype with phenotype in contrasting environments. The Plant Journal 97:19-39. doi: $10.1111 /$ tpj. 14113

[32] Rida Fatima Ahmed, Muhammad Irfan, Hafiz Abdullah Shakir, Muhammad Khan \& Lijing Chen, 2020. Engineering drought tolerance in plants by modification of transcription and signalling factors. Biotechnology \& Biotechnological Equipment, 34(1):781-789, https://doi.org/10.1080/13102818.2020.1805359

[33]Ruben Rufo, Silvio Salvi, Conxita Royo and Jose Miguel Soriano, 2020. Exploring the Genetic Architecture of Root-Related Traits in Mediterranean Bread Wheat Landraces by Genome-Wide Association Analysis. Agronomy10:613doi:10.3390/agronomy10050613

[34] Sajeevan R.S., Parvathi M.S. and Nataraja K.N., 2017. Leaf wax trait in crops for drought and biotic stress tolerance: regulators of epicuticular wax synthesis and role of small RNAs. Ind J plant physio. 22,434-447 https://doi.org/10.1007/s40502-017-0333-9

[35] Salah E. Abdel-Ghany, Fahad Ullah, Asa Ben-Hur and Anireddy S. N. Reddy, 2020. Transcriptome Analysis of Drought-Resistant and Drought-Sensitive Sorghum (Sorghum bicolor) Genotypes in Response to PEG-Induced Drought Stress, International Journal of Molecular Sciences https://doi.org/10.3390/ijms2103077

[36] Sharmila Polumahanthi, Sarada M ani N., Sudhakar Pola, Dora S.V.V.S.N. and Nageswara Rao S., 2014. Tissue Culture, Molecular and Genetic Approaches to Sorghum Crop Improvement - A Review. Indian Journal of Plant Sciences Vol.4 Pp.97-113.

[37] Shavrukov Y, Kurishbayev A, Jatayev S, Shvidchenko V, Zotova L, Koekemoer F, de Groot S, Soole K and Langridge P, 2017. Early Flowering as a Drought Escape Mechanism in Plants: How Can It Aid Wheat Production?. Front. Plant Sci. 8:1950. doi: 10.3389/fpls.2017.01950

[38] Tesfamichael Abraha, Stephen Mwangi Githiri, Remmy Kasili, Woldeamlak Araia, Aggrey Bernard Nyende, 2015. Genetic Variation among Sorghum (Sorghum bicolor L. Moench) Landraces from Eritrea under Post-Flowering Drought Stress Conditions. American Journal of Plant Sciences, 6, 1410-1424. http://dx.doi.org/10.4236/ajps.2015.69141

[39] Tuinstra, M.R., Grote, E.M., Goldbrough, P.M., and Ejeta, G. (1997). Genetic analysis of post-flowering drought tolerance and components of grain development in Sorghum bicolor L. Moench. Molecular Breeding 3: 439-448. 
[40] Varoquauxa Nelle, Benjamin Cole, Cheng Gao, Grady Pierroz, Christopher R. Baker, Dhruv Patel, Mary Madera, Tim Jeffers, Joy Hollingsworth, Julie Sievert, Yuko Yoshinaga, Judith A. Owiti, Vasanth R. Singan, Stephanie DeGraaf, Ling Xu, Matthew J. Blow, Maria J. Harrison, Axel Visel, Christer Jansson, Krishna K. Niyogi, Robert Hutmacher, Devin Coleman-Derr, Ronan C. O’Malley, John W. Taylor, Jeffery Dahlberg, John P. Vogel, Peggy G. Lemaux, and Elizabeth Purdom, 2019. Transcriptomic analysis of fielddroughted sorghum from seedling to maturity reveals biotic and metabolic responses. Proceeding of the National Academy of Sciences of the United State of America 116 (52): 27124-27132 https://doi.org/10.1073/pnas.1907500116

[41] Velazco JG, Jordan DR, Mace ES, Hunt CH, Malosetti M and van Eeuwijk FA, 2019. Genomic Prediction of Grain Yield and Drought-Adaptation Capacity in Sorghum Is Enhanced by Multi-Trait Analysis. Front. Plant Sci 10:997. doi:10.3389/fpls.2019.00997

[42] Xiong, L., Wang, R., Mao, G., and Koezan, J. (2006). Identification of drought tolerance determinants by genetic analysis of root responses to drought stress and abscisic acid. Plant Physiologt 142: 1065-1074.

[43] Xue D, Zhang X, Lu X, Chen G and Chen Z-H, 2017. Molecular and Evolutionary Mechanisms of Cuticular Wax for Plant Drought Tolerance. Front. Plant Sci. 8:621. doi: 10.3389/fpls.2017.00621

[44]Zhang Deng-feng, ZENG Ting-ru, LIU Xu-yang, GAO Chen-xi, LI Yong-xiang, LI Chun-hui, SONG Yanchun, SHI Yun-su, WANG Tian-yu, LI Yu, 2019. Transcriptomic profiling of sorghum leaves and roots responsive to drought stress at the seedling stage. Journal of Integrative Agriculture, 18(9): 1980-1995.

Citation: Tamirat Bejiga,et.al., (2021). "Molecular Breeding Approaches for Drought and Drought Related Traits in Sorghum: A Review Article”, International Journal of Research Studies in Agricultural Sciences (IJRSAS), 7(7), pp. 23-34 DOI: http://dx.doi.org/10.20431/2454-6224.0707003

Copyright: (C) 2021 Authors. This is an open-access article distributed under the terms of the Creative Commons Attribution License, which permits unrestricted use, distribution, and reproduction in any medium, provided the original author and source are credited. 